\title{
Asymmetric parametric amplification in nonlinear left-handed transmission lines
}

\author{
David A. Powell, ${ }^{\text {a) }}$ llya V. Shadrivov, and Yuri S. Kivshar \\ Nonlinear Physics Centre, Research School of Physics and Engineering, Australian National University, \\ Canberra, Australian Capital Territory 0200, Australia
}

(Received 15 December 2008; accepted 8 February 2009; published online 27 February 2009)

\begin{abstract}
We study parametric amplification in nonlinear left-handed transmission lines, which serve as model systems for nonlinear negative-index metamaterials. We experimentally demonstrate the amplification of a weak signal in the three following regimes: with the signal in the left-handed band, with the signal in the stop band, and with the signal at a defect frequency. In particular, we demonstrate the amplification of the incident wave by up to $15 \mathrm{~dB}$ in the left-handed regime. () 2009 American Institute of Physics. [DOI: 10.1063/1.3089842]
\end{abstract}

Left-handed transmission lines are compact systems showing regimes of backward wave propagation similar to negative-index metamaterials. They have been applied to a number of engineering applications, including the study of leaky wave antennas, compact resonators, and dual-band couplers (see, e.g., Ref. 1 and references therein). In a number of these applications, nonlinear elements have been introduced to create tunable structures ${ }^{2,3}$ and, in addition, they have been used as a platform for the study of nonlinear wave propagation in the systems supporting the propagation of backward waves. $^{4-6}$

Parametric gain is a nonlinear process whereby a highenergy pump wave exchanges energy with a weaker signal wave through modulation of the material or circuit parameters, thus amplifying it. This effect is commonly used in optical systems, and it has been proposed as a way to mitigate the losses in negative-index metamaterials. ${ }^{7}$ In particular, in Ref. 8, parametric gain was exhibited in the lefthanded region for a short structure consisting of seven periods with an external dc bias, and parametric generation of backward waves in similar transmission lines was studied both experimentally and theoretically in Ref. 9.

Recently, we have demonstrated a bistable regime of the left-handed propagation in an asymmetric nonlinear lefthanded transmission line due to the existence of multiple dynamic states. ${ }^{10}$ In this letter, we demonstrate that such structures may exhibit substantial parametric gain in three different regimes of the signal wave: with the signal in the left-handed band, with the signal in the stop band, and with the signal at a defect frequency. In particular, we demonstrate the amplification of the incident wave by up to $15 \mathrm{~dB}$ in the left-handed regime.

Our nonlinear transmission line consists of 20 periods of elementary cells, each cell containing a series varactor diode (SMV1405), shunt inductor (18 $\mathrm{nH}$ chip-type), and a $10 \mathrm{~mm}$ section of $50 \Omega$ microstrip transmission line, as shown in Fig. 1. The linear transmission response is represented by a solid curve in Fig. 2. The dispersion relation is of great importance to all parametric processes, and it can be calculated analytically as ${ }^{1}$

${ }^{a)}$ Electronic mail: david.a.powell@anu.edu.au.

$$
\cos \kappa=\left[1-\frac{1}{2}\left(\omega L_{R}-\frac{1}{\omega C_{L}}\right)\left(\omega C_{R}-\frac{1}{\omega L_{L}}\right)\right]
$$

where $\kappa$ is the Bloch wave number (in radians per period), $L_{L}$ and $C_{L}$ are the shunt inductance and series capacitance introduced to create the left-handed line; $C_{R}=d / Z_{0} v$ and $L_{R}=Z_{0} d / v$ are the equivalent shunt capacitance and series inductance of the microstrip line having impedance $Z_{0}$ and the propagation velocity $v$, calculated under the assumption that each section length $d$ is short compared to the wavelength. Such structures demonstrate a transmission spectrum with a low-frequency stop band, a left-handed pass band, where the phase velocity and Poynting vector are in opposite directions, another stop band (which may be absent if the left- and right-handed band edges coincide), and a righthanded pass band where the phase velocity and Poynting vector are codirectional.

We first verify the dispersion of the transmission line experimentally. One of the standard approaches is to scan the fields above the structure for each frequency and perform a Fourier transform in the spatial domain in order to find the dominant spatial frequencies. However, for a structure such as ours which is only 20 periods long, this approach gives a very poor resolution of spatial frequencies. Instead, we make the $a$ priori assumption that only a single Bloch mode propagates in the structure at any given frequency. We then perform an optimization procedure to find its wave number, as well as the amplitudes of the forward and backward propagating waves, using the procedure described in Ref. 11. Importantly, our procedure allows retrieval of both the real and imaginary parts of the wave number. The latter characterizes the decay length of the waves inside and outside of the band gaps. The corresponding results are shown in Fig. 3, and they are compared with Eq. (1), with the parasitic inductance of the diode added to the term $L_{R}$.

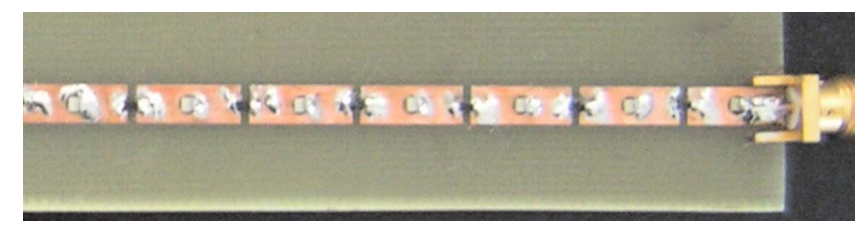

FIG. 1. (Color online) Photograph showing six periods of the nonlinear left-handed transmission line used in our experiments. 


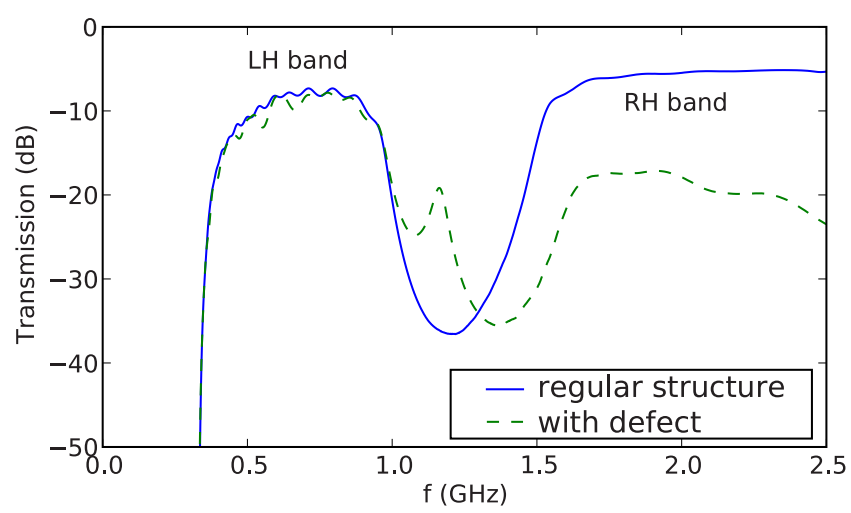

FIG. 2. (Color online) Transmission response of the left-handed transmission line before and after the inclusion of a defect.

It can be seen that the agreement is quite good over the whole frequency range, and that the locations of the band edges are also in agreement with the theory. Some additional imaginary parts of the wave number in the transmission bands are seen in the experiment due to the losses which are not included in the simplified circuit model. The relative amplitudes of the forward and backward Bloch modes (not shown) verify the regions of the forward and backward wave propagation. For high input powers we do not observe any smooth shift from this dispersion curve, instead artifacts appear as the wave no longer satisfies the Bloch condition, and the dispersion curve becomes meaningless.

In order to achieve strong parametric amplification, it is necessary to ensure that the generated signal adds to itself constructively over the length of the structure. In the three-frequency parametric processes with all components forward propagating, this will occur when $\omega_{1}+\omega_{2}=\omega_{3}$ and $\kappa_{1}+\kappa_{2}=\kappa_{3}$. For any frequency component where the backward wave is dominant a negative sign should appear in front of $\kappa$. Here subscript 1 indicates the signal wave, subscript 3 indicates the pump wave, and subscript 2 indicates the idler wave, which is automatically generated through the parametric process. However, it should be noted that for relatively short structures with strong nonlinear interaction and reflection from the structure edges such as our transmission line, parametric amplification can occur which satisfies different conditions for the wave numbers. ${ }^{12}$ These conditions may depend on the length of the structure and reflections from the boundaries.

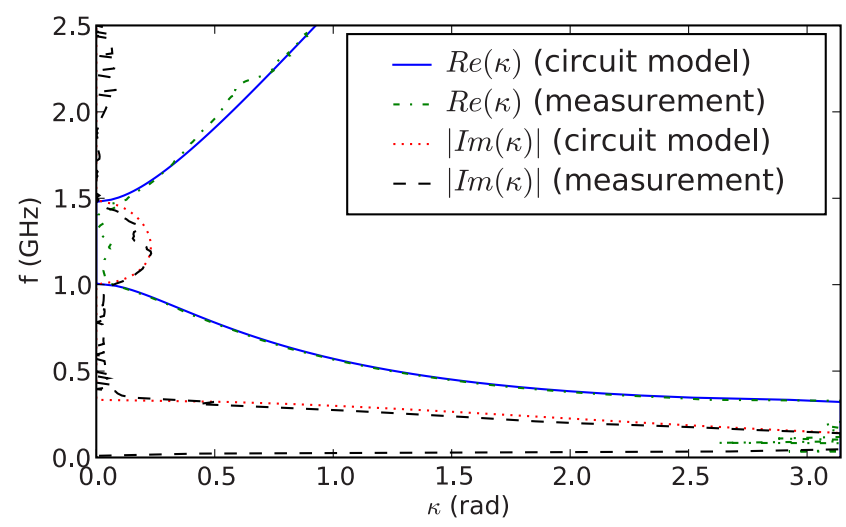

FIG. 3. (Color online) Dispersion curves fitted to the experimental data using the retrieval procedure in Ref. 11 and from the circuit theory. (a) Forward orientation

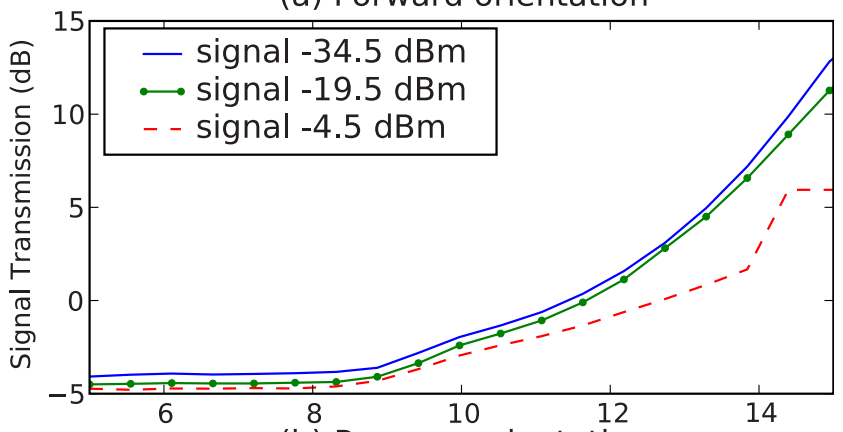

(b) Reverse orientation

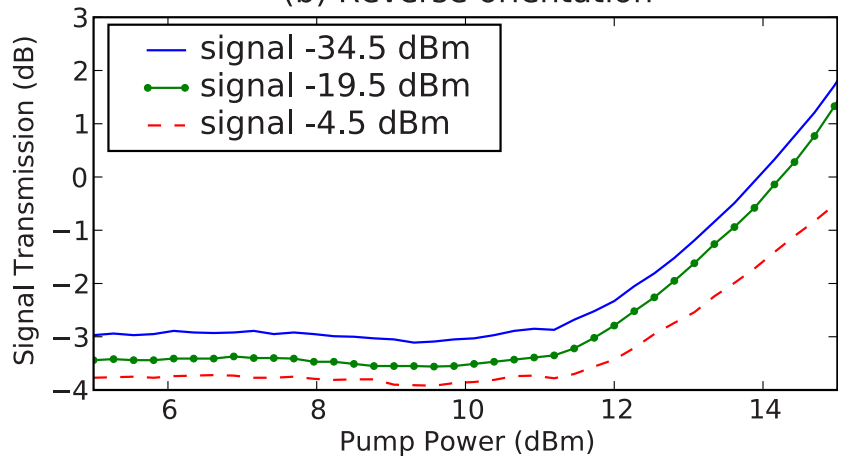

FIG. 4. (Color online) Gain vs pump power for the signal in the left-handed band for two different orientations of the transmission line.

In order to measure parametric gain, the signal and the pump are combined with a $3 \mathrm{~dB}$ combiner. Since the combiner does not offer good isolation over all frequencies, a $10 \mathrm{~dB}$ attenuator is applied to the signal line to provide isolation of the source generator from the pump signal. The pump and signal frequencies are determined empirically to find the frequencies of the maximum gain. The pump is applied at $f_{3}=1.43 \mathrm{GHz}$, which is inside the stop band and thus has $\kappa_{3}=0$. The signal is applied in the left-handed band at $f_{1}=806 \mathrm{MHz}$ with $\kappa_{1}=-0.45 \mathrm{rad}$, and it is codirectional to the excited idler with $f_{2}=624 \mathrm{MHz}$ and $\kappa_{2}=-0.85 \mathrm{rad}$.

Gain is measured by taking the ratio of the signal power at the output of the transmission line to the signal power incident at the input, thus it also includes the effects of the losses in the line. Figure 4(a) shows the gain of the transmission line as a function of the pump power incident at the input port of the transmission line. At low powers the pump has essentially no effect, and the attenuation of 4-5 dB due to the loss through the transmission line is observed. Once the pump power reaches the value of $12 \mathrm{dBm}$ it can be seen that the pump compensates for the losses of the line, and subsequently the system provides the gain up to $15 \mathrm{~dB}$ for pump powers of $15 \mathrm{dBm}$. Beyond this point the system becomes a parametric generator. Also shown is the influence of the signal power, which reduces the gain and for very high input powers the gain process is not so clearly observable due to the interaction with the many intermodulation products present in the system.

In this structure, there is only a single diode per unit cell, and they are all identically aligned, thus there is a strong asymmetry in the system response. This asymmetry strongly affects the parametric gain, and when both the signal and the pump are applied in the other port of the transmission line, the parametric gain is much reduced, as shown in Fig. 4(b). This may be due to the onset of the forward conduction, or 


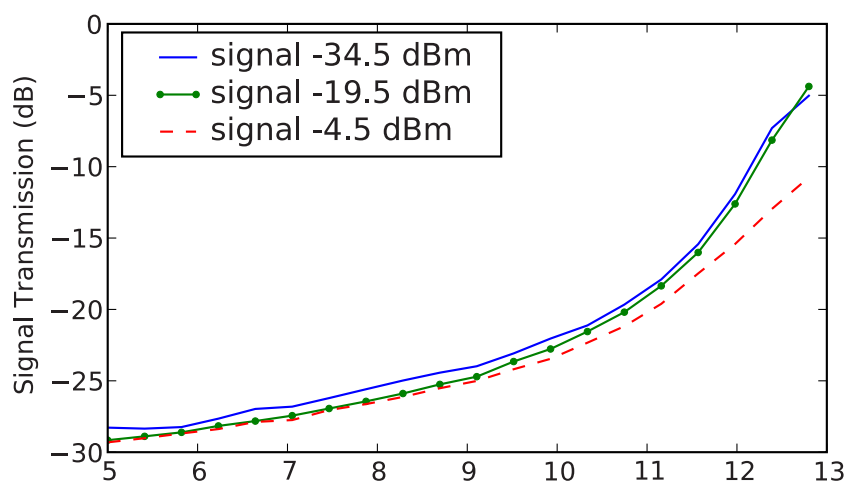

FIG. 5. (Color online) Gain vs pump power for the signal within the band gap.

the asymmetry of the capacitance-voltage relationship of the diodes.

We also performed a thorough investigation of possible regimes of the parametric gain. We were not able to find any parametric processes with the signal operating in the righthanded band. This is not unexpected, since at these frequencies the series capacitance acts as a very small impedance, and has little ability to modulate the propagating wave. However, it was found that a signal within the stop band could be amplified by a pump in the right-handed band. This is shown in Fig. 5, where the pump was at $f_{3}=1.958 \mathrm{GHz}$, $k_{3}=0.46 \mathrm{rad}$, the signal at $f_{1}=1.376 \mathrm{GHz}, \kappa_{1}=0$, and the idler at $f_{2}=582 \mathrm{MHz}, \kappa_{2}=-0.97 \mathrm{rad}$.

Finally, we consider the effects associated with the introduction of a defect into the transmission line. Defects within a periodic system have the ability to localize waves, and they are often used to create resonant cavities at frequencies inside the band gaps. In our case, the defect is created by introducing an additional inductor in series with the diode. This creates a defect mode well within the stop band, as well as a degradation of the transmission in the right-handed band, as shown by the dashed curve in Fig. 2. A field scan was performed (not shown) to demonstrate that the fields at this frequency are localized on the defect site, and that they decay strongly with distance.

Parametric amplification is performed with a signal at the defect with $f_{1}=1.164 \mathrm{GHz}, \kappa_{1}=0$, idler in the lefthanded band with $f_{2}=730 \mathrm{MHz}, \kappa_{2}=-0.60 \mathrm{rad}$, and pump in the right-handed band at $f_{3}=1.893 \mathrm{GHz}, \kappa_{1}=0.41 \mathrm{rad}$, and the results are summarized in Fig. 6. It can be seen that the gain is much lower and never becomes positive, which can be attributed to the defect cell strongly reflecting the signal in the right-handed band, thus inhibiting transmission of the pump.

In conclusion, we have experimentally studied parametric amplification and generation in nonlinear left-handed

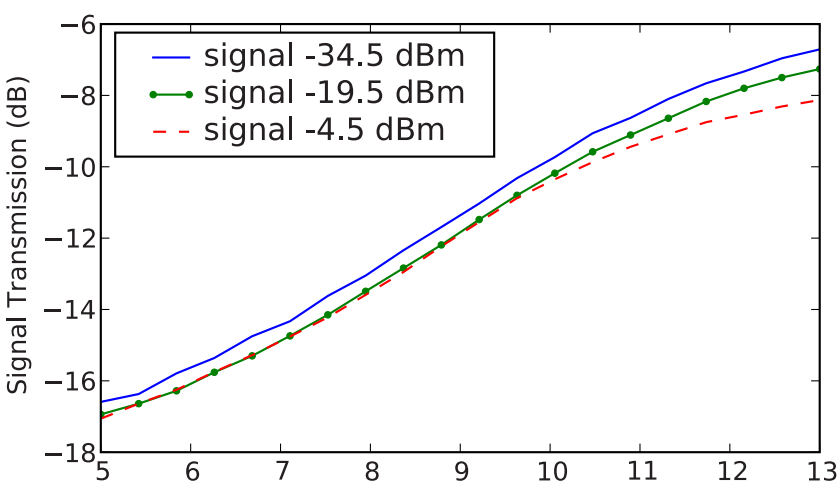

FIG. 6. (Color online) Gain vs pump power for the signal at the frequency of the defect mode.

transmission lines. By utilizing the complex dispersion characteristics of this system, we have demonstrated the amplification of a weak signal in the three different regimes: with the signal in the left-handed propagation band, with the signal in the stop band, and with the signal at a defect frequency. By engineering the dispersion of such structures, it should be possible to further enhance the gain which can be produced, for example, by shifting the pump frequency to the band edge, where the real wave number is still zero, but the transmission is much higher. Our results show that substantial gain can be achieved in nonlinear systems exhibiting backward wave propagation, and they confirm that parametric processes are promising candidates for mitigating the losses found in artificial backward wave media, including negative-index metamaterials.

The authors acknowledge the financial support of the Australian Research Council.

${ }^{1}$ A. Lai, T. Itoh, and C. Caloz, IEEE Microw. Mag. 5, 34 (2004).

${ }^{2}$ I. Gil, J. Bonache, J. Garcia-Garcia, and F. Martin, IEEE Trans. Microwave Theory Tech. 54, 2665 (2006).

${ }^{3}$ D. Kuylenstierna, A. Vorobiev, P. Linner, and S. Gevorgian, IEEE Microw. Wirel. Compon. Lett. 16, 167 (2006).

${ }^{4}$ A. B. Kozyrev, H. Kim, A. Karbassi, and D. W. van der Weide, Appl. Phys. Lett. 87, 121109 (2005).

${ }^{5}$ A. B. Kozyrev and D. W. van der Weide, Appl. Phys. Lett. 91, 254111 (2007)

${ }^{6}$ A. B. Kozyrev and D. W. van der Weide, J. Phys. D 41, 173001 (2008).

${ }^{7}$ A. K. Popov and V. M. Shalaev, Appl. Phys. B 84, 131 (2006).

${ }^{8}$ A. Kozyrev, H. Kim, and D. van der Weide, Appl. Phys. Lett. 88, 264101 (2006).

${ }^{9}$ A. S. Gorshkov, G. A. Lyakhov, K. I. Voliak, and L. A. Yarovoi, Physica D 122, 161 (1998).

${ }^{10}$ D. A. Powell, I. V. Shadrivov, and Yu. S. Kivshar, Appl. Phys. Lett. 92, 264104 (2008).

${ }^{11}$ A. A. Sukhorukov, S. Ha, I. V. Shadrivov, D. A. Powell, and Yu. S. Kivshar, arXiv:0901.3244v1; http://arxiv.org/abs/0901.3244.

${ }^{12}$ Y. R. Shen, The Principles of Nonlinear Optics (Wiley, New York, 1984). 